\title{
Revista Colombiana de

\section{Corrección quirúrgica exitosa de ruptura de aneurisma del seno de Valsalva Sakakibara tipo IV y cierre de comunicación interventricular perimembranosa}

\author{
Dulman O. Pineda-De Paz ${ }^{a, *}$, Jorge E. Hernández-del Río ${ }^{a}$, \\ Christian González-Padilla ${ }^{a}$, David Ramírez-Cedillo ${ }^{\mathrm{b}}$ \\ e Italo D. Masini-Aguilera ${ }^{\mathrm{b}}$
}

\author{
a Departamento de Cardiología. Antiguo Hospital Civil de Guadalajara "Fray Antonio Alcalde", Universidad de Guadalajara, \\ Ciudad de Guadalajara, Jalisco, México \\ b Departamento de Cirugía Cardiovascular. Antiguo Hospital Civil de Guadalajara "Fray Antonio Alcalde", Universidad de \\ Guadalajara, Ciudad de Guadalajara, Jalisco, México
}

Recibido el 30 de junio de 2017; aceptado el 22 de marzo de 2018

Disponible en Internet el 2 de junio de 2018

\section{PALABRAS CLAVE \\ Seno de Valsalva; \\ Aneurisma; \\ Ecocardiograma; \\ Congénito}

\section{KEYWORDS}

Sinus of Valsalva; Aneurysm; Echocardiogram; Congenital

\begin{abstract}
Resumen El aneurisma del seno de Valsalva es una anomalía cardíaca que puede ser congénita o adquirida. Es referido como una anomalía congénita rara, usualmente asintomática detectada en un ecocardiograma 2D de rutina, hasta presentaciones relacionadas con la compresión de estructuras adyacentes o como cortocircuitos intracardíacos causados por la ruptura de un aneurisma del seno de Valsalva dentro de una cavidad derecha del corazón. Se expone el caso de un paciente de sexo masculino, de 27 años de edad, que acudió al departamento de emergencia con disnea progresiva hasta ser de pequeños esfuerzos a quien se le documentó ruptura de aneurisma del seno de Valsalva no coronariano, clasificación de Sakakibara tipo IV, asociado a comunicación interventricular perimembranosa. Se hizo cirugía cardíaca para tratamiento quirúrgico definitivo, el cual fue exitoso. Se hace una revisión breve de la literatura acerca de esta patología, su definición, etiología, epidemiología, clasificación, diagnóstico y tratamiento. (c) 2018 Sociedad Colombiana de Cardiología y Cirugía Cardiovascular. Publicado por Elsevier España, S.L.U. Este es un artículo Open Access bajo la licencia CC BY-NC-ND (http:// creativecommons.org/licenses/by-nc-nd/4.0/).
\end{abstract}

Successful surgical correction of a rupture of a Sakakibara type IV Valsalva sinus aneurysm and closure of the perimembranous ventricular septal defect

Abstract An aneurysm of the sinus of Valsalva is a cardiac anomaly that can be congenital or acquired. It is referred to as a rare congenital anomaly, usually asymptomatic and detected in a routine 2-D echocardiogram. It is also found associated with the compression of adjacent

\footnotetext{
* Autor para correspondencia.

Correo electrónico: dulmanpinedamd@gmail.com (D.O. Pineda-De Paz).
} 
structures or as intra-cardiac short-circuits caused by the rupture of an aneurysm of the sinus of Valsalva within a right cavity of the heart. The case is presented of a 27 year-old male, who arrived in the Emergency Department with progressive dyspnoea even with small exertions and was diagnosed with rupture of a Sakakibara type IV, non-coronary sinus of Valsalva aneurysm, associated with a perimembranous ventricular septal defect. Open heart surgery was performed for definitive surgical treatment, which was successful. A short review of the literature is presented on this condition, its definition, aetiology, epidemiology, classification, diagnosis, and treatment.

(C) 2018 Sociedad Colombiana de Cardiología y Cirugía Cardiovascular. Published by Elsevier España, S.L.U. This is an open access article under the CC BY-NC-ND license (http:// creativecommons.org/licenses/by-nc-nd/4.0/).

\section{Caso}

Paciente masculino, de 27 años de edad, sin factores de riesgo cardiovascular y sin antecedentes médicos de importancia, quien fue referido al departamento de urgencias por presentar disnea progresiva de pequeños esfuerzos de una semana de evolución. Comenzó con su padecimiento actual un mes previo con disnea a grandes esfuerzos que rápidamente progresó a clase funcional NYHA IV. El examen físico cardiovascular al momento del ingreso reveló ingurgitación venosa yugular y descenso de $x$. no visibles, levantamiento sistólico paraesternal izquierdo palpable, impulso apical visible y palpable en el séptimo espacio intercostal en línea axilar anterior, de $2 \mathrm{~cm}$ de diámetro, no sostenido; ruidos cardiacos rítmicos, S1 borrado por soplo holosistólico rudo, de intensidad IV/VI con thrill, con epicentro en borde paraesternal izquierdo e irradiación en banda, S2 con reforzamiento del componente P2; no se auscultó S3 ni S4.

El electrocardiograma basal reveló ritmo sinusal, frecuencia cardíaca en $85 \mathrm{lpm}$, eje -90 , con crecimiento biauricular y crecimiento ventricular derecho, bloqueo incompleto de rama derecha del haz de His y bloqueo del fascículo anterior de la rama izquierda del haz de His.

La radiografía de tórax reveló ensanchamiento mediastinal, principalmente a expensas de la arteria pulmonar e interlobar prominente, cardiomegalia global significativa, con ICT 0,67 y datos sugestivos de crecimiento importante de la aurícula derecha (fig. 1).

El ecocardiograma transtorácico reportó fracción de eyección del ventrículo izquierdo del $60 \%$, ventrículo izquierdo con movilidad normal en todos sus segmentos y función diastólica normal; se observó comunicación interventricular, tipo subaórtico de $13,6 \mathrm{~mm}$ con gradiente máximo transdefecto de $51 \mathrm{~mm} \mathrm{Hg}$ y flujo de izquierda a derecha. QP/QS 1.54. Hipertensión pulmonar probable de grado moderado con presión sistólica de la arteria pulmonar (PSAP) en $59 \mathrm{~mm} \mathrm{Hg}$, estimada a través del gradiente máximo transdefecto del septum interventricular. En el eje corto paraesternal a nivel de grandes vasos se observó válvula aórtica trivalva y aneurisma del seno de Valsalva no coronariano con ruptura hacia la aurícula derecha, evidenciándose 2 jets en el doppler color; uno en dirección a la pared libre de la aurícula derecha y otro con dirección a la valva septal de la válvula tricúspide, con un gradiente

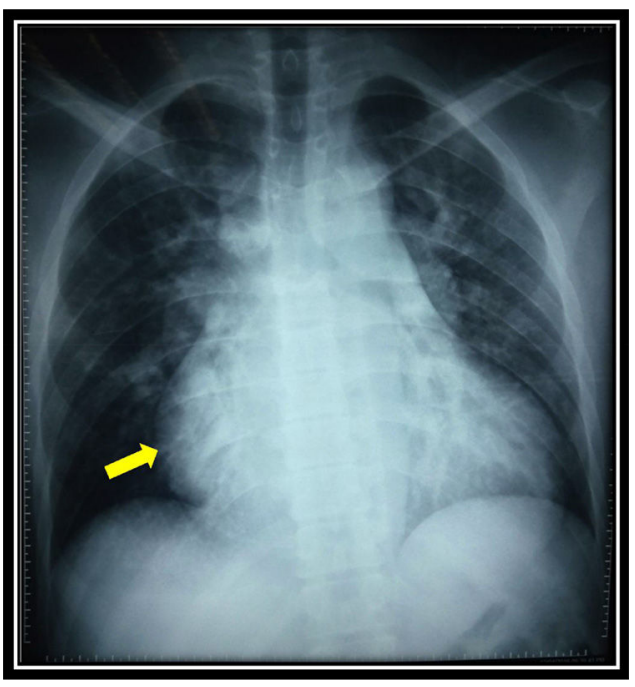

Figura 1 Radiografía de tórax en proyección postero-anterior que muestra ensanchamiento mediastinal, principalmente a expensas del arco de la arteria pulmonar prominente, cardiomegalia significativa global con ICT 0,67 con datos sugestivos de considerable crecimiento auricular derecho (flecha).

máximo de $58 \mathrm{~mm} \mathrm{Hg}$, clasificación de Sakakibara tipo IV (figs. $2 \mathrm{~A}$ y $\mathrm{B}$ ).

Fue llevado a cirugía cardíaca durante la cual se halló válvula aórtica trivalva, ruptura lineal en valva no coronariana y aneurisma de seno de Valsalva no coronariano roto de $5 \mathrm{~mm}$ hacia aurícula derecha: Se corrigió el aneurisma con parche de dacrón y pledget de pericardio más reparación de la valva no coronariana (figs. 3 y 4 ).

Además, se encontró comunicación interventricular de $10 \mathrm{~mm}$ tipo perimembranosa, así que se realizó cierre de dicho defecto con puntos separados con pledget de teflón y parche de doble velour. Cirugía sin complicaciones, con tiempo adecuado de bomba de circulación extracorpórea (104 minutos) y pinzado aórtico de 83 minutos.

\section{Discusión}

El aneurisma del seno de Valsalva es una anomalía cardíaca que puede ser congénita o adquirida. Thurnam fue 

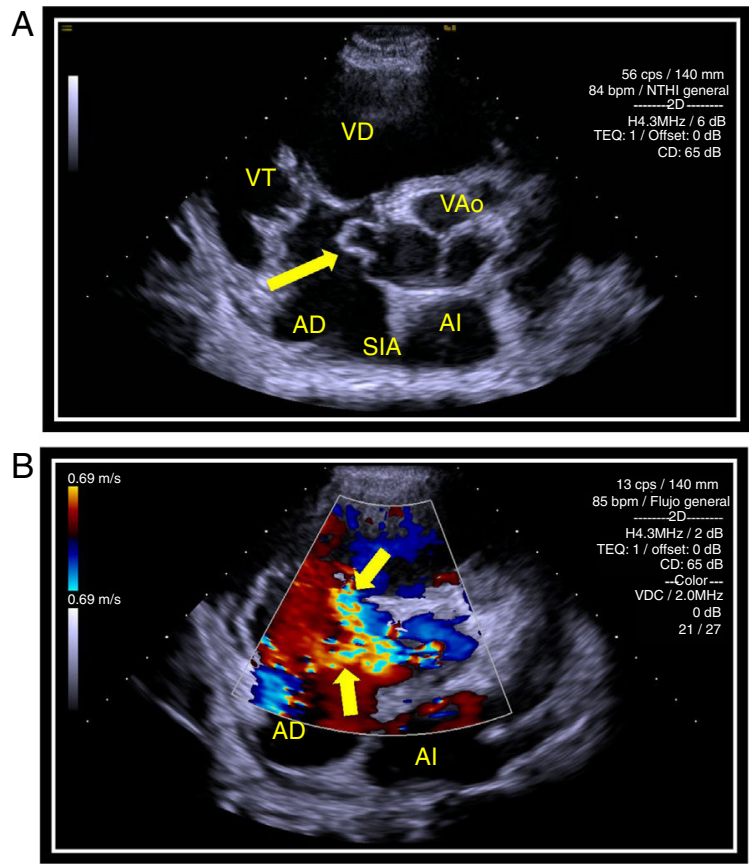

Figura 2 Panel A Ecocardiograma transtorácico en 2D, eje corto paraesternal a nivel de los grandes vasos, muestra válvula aórtica trivalva con presencia de aneurisma del seno de Valsalva no coronariano (flecha). Panel B Ecocardiograma transtorácico en $2 \mathrm{D}$, eje corto paraesternal a nivel de los grandes vasos, con doppler color en donde se observan 2 jets, uno en dirección a la pared libre de la aurícula derecha (flecha inferior) y otro con dirección a la valva septal de la válvula tricúspide (flecha superior), con un gradiente máximo de $58 \mathrm{~mm} \mathrm{Hg}$, compatible con ruptura de aneurisma del seno de Valsalva no coronariano, clasificación de Sakakibara tipo IV.

VD: ventrículo derecho; VT: válvula tricúspide; $A D$ : aurícula derecha; VAo: válvula aórtica; SIA: septum interauricular; AI: aurícula izquierda.

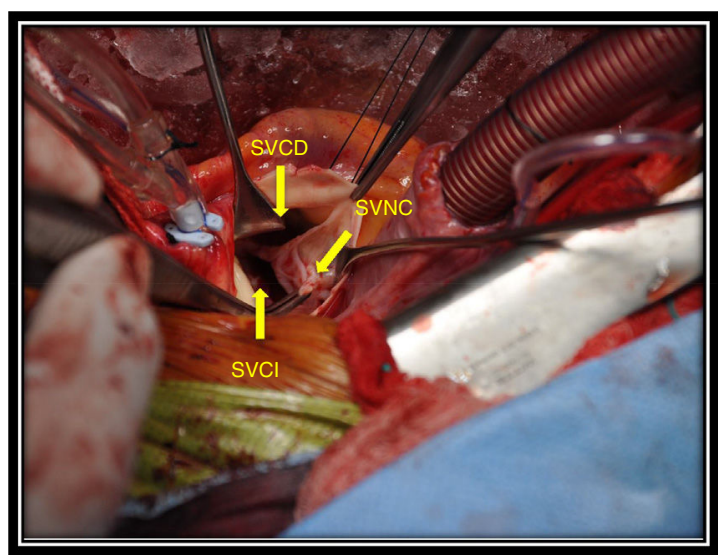

Figura 3 Cirugía cardíaca en la que se observa la válvula aórtica con sus tres senos de Valsalva: coronariano derecho (SVCD), coronariano izquierdo ( $\mathrm{SVCl}$ ) y no coronariano (SVNC); en éste último destaca la presencia de un aneurisma roto hacia la aurícula derecha, previo a su reparación.

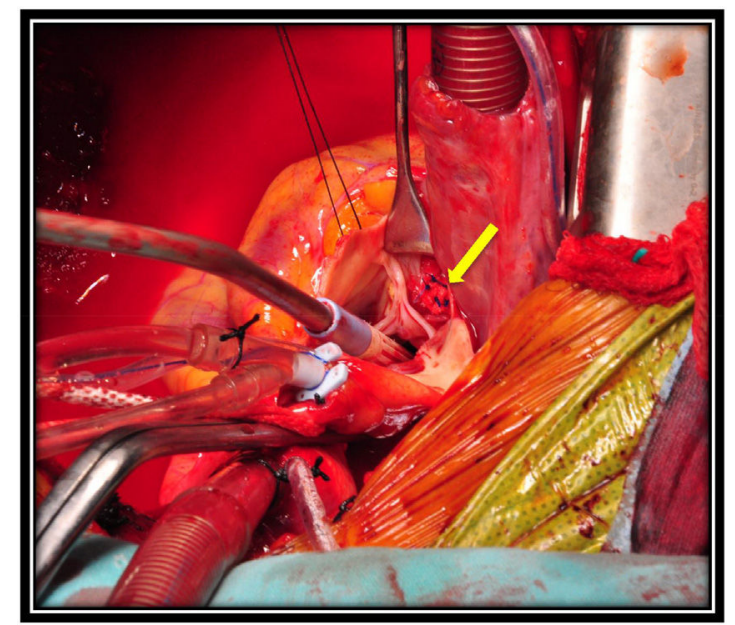

Figura 4 Cirugía a corazón abierto en la que se observa el seno de Valsalva no coronariano reparado con un parche de pledget de pericardio (flecha).

Tabla 1 Clasificación para los aneurismas del seno de Valsalva propuesta por Sakakibara y Konno (año, 1962)

Tipo I Conecta el seno de Valsalva derecho y el tracto de salida del ventrículo derecho debajo de la válvula pulmonar Tipo II Conecta el seno de Valsalva derecho y el ventrículo derecho en la crista supraventricularis

Tipo Illa Conecta el seno de Valsalva derecho y la aurícula derecha

Tipo IIlv Conecta la zona posterior del seno de Valsalva derecho y el ventrículo derecho

Tipo III a + v Conecta el seno de Valsalva derecho y ambos, aurícula derecha y ventrículo derecho

Tipo IV Conecta el seno de Valsalva no coronario y la aurícula derecha

el primero en describir esta anomalía en 1840; posteriormente, Hope lo describió en 1939. Usualmente es referido como una anomalía congénita rara. Los aneurismas del seno de Valsalva congénitos son generalmente silentes desde el punto de vista clínico, pero pueden variar desde una dilatación asintomática detectada en un ecocardiograma $2 \mathrm{D}$ de rutina, hasta presentaciones relacionadas con la compresión de estructuras adyacentes o como cortocircuitos intracardiacos causados por la ruptura de un aneurisma del seno de Valsalva dentro de una cámara derecha del corazón ${ }^{1}$.

El aneurisma del seno de Valsalva se ha encontrado en $0,09 \%$ en grandes series de autopsias en los Estados Unidos. Aproximadamente, $65-85 \%$ de los aneurismas se originan en el seno derecho, el 10 al $30 \%$ en el seno no coronariano y solamente $<5 \%$ se originan en el seno coronariano izquierdo ${ }^{2,3}$. El $10 \%$ de las rupturas generan un cortocircuito que comunica el seno de Valsalva aneurismático con la aurícula derecha ${ }^{1}$. Fueron Sakakibara y Konno quienes propusieron una clasificación de acuerdo con dos factores: el seno coronariano de origen del aneurisma y la cavidad cardíaca con la que comunica el cortocircuito a la hora de romperse $^{4}$ (tabla 1).

El ecocardiograma 2 D es altamente eficaz para detectar los aneurismas del seno de Valsalva; sin embargo, en algunos 
estudios se insiste en el incremento de la superioridad del ecocardiograma $3 \mathrm{D}^{5}$.

De manera convencional, con una mortalidad de menos del $2 \%$, la reparación quirúrgica de la ruptura del aneurisma del seno de Valsalva ha sido el estándar de oro para el tratamiento definitivo. Se recomienda la cirugía urgente en todos los pacientes con ruptura del aneurisma del seno de Valsalva, especialmente en aquellos con cortocircuitos intracardiacos ya que sin cirugía la mortalidad es cercana al 100\% al año del diagnóstico ${ }^{6-8}$.

Se presenta el caso de un paciente joven sin factores de riesgo cardiovascular y sin ninguna condición cardíaca o extracardíaca predisponente para aneurisma del seno de Valsalva de causa adquirida, por lo cual se concluye que el aneurisma es congénito y que, como se explica en la literatura, la presentación sintomática se debe a la ruptura del mismo hacia una cavidad cardíaca derecha, en este caso hacia la aurícula derecha, de modo que es posible clasificarlo según la propuesta de Sakakibara y Konno en tipo $I^{5}$. Resultó interesante reportar este caso debido a que la literatura menciona la poca frecuencia con que se presentan los aneurismas del seno de Valsalva. Entre ellos los más comunes son los que comprometen el seno de Valsalva coronariano derecho y sólo el $10 \%$ se rompe hacia la aurícula derecha, como ocurrió en este caso; así mismo, se asoció a comunicación interventricular subaórtica, pero ambos defectos fueron resueltos de manera satisfactoria mediante cirugía correctiva urgente. El paciente egresó con control ecocardiográfico sin demostrar cortocircuitos posteriores y permanece asintomático cardiovascular en las consultas de seguimiento.

\section{Conclusión}

El aneurisma del seno de Valsalva es una anomalía cardíaca congénita o adquirida; la primera forma suele darse en sujetos jóvenes, quienes pueden persistir asintomáticos hasta el momento en que ocurra la ruptura del mismo hacia una cavidad derecha del corazón. El diagnóstico de la ruptura del seno de Valsalva debe hacerse con prontitud. El tratamiento más efectivo lo constituye la corrección quirúrgica del defecto de manera oportuna para mejorar el pronóstico de los pacientes.

\section{Financiación}

Ninguna.

\section{Conflicto de intereses}

Ninguno.

\section{Bibliografía}

1. Ring WS. Congenital Heart Surgery Nomenclature and Database Project: Aortic Aneurysm Sinus of Valsalva Aneurysm, and Aortic Dissection. Ann Thorac Surg. 2000;69:S147-63.

2. Meier JH, Seward JB, Miller FA, Oh JK, Enriquez M. Aneurysms in the left ventricular outflow tract: clinical presentation, causes, and echocardiographic features. J Am Soc Echocardiogr. 1998;11:729-45.

3. Prian GW, Diethrich EB. Sinus of Valsalva abnormalities. A specific differentiation between aneurysms of an aneurysms involving the sinuses of Valsalva. Vasc Surg. 1973;7:155-64.

4. Xin-jin L, Xuan L, Bo P, Hong-wei G, Wei W, Shou-jun L, et al. Modified Sakakibara classification system for ruptured sinus of Valsalva aneurysm. J Thorac Cardiovasc Surg [Internet]. Elsevier Inc.;. 2013;146:874-8. Disponible en: https://doi.org/10.1016/j.jtcvs.2012.12.059.

5. Raslan S, Nanda NC, Lloyd L, Khairnar P, Reilly SD, Holman WL. Incremental value of live/real time three-dimensional transesophageal echocardiography over the two-dimensional technique in the assessment of sinus of Valsalva aneurysm rupture. Echocardiography. 2011;28:918-20.

6. Flynn MS, Castello R, McBride LW, Labovitz AJ. Ruptured congenital aneurysm of the sinus of Valsalva with persistent left superior vena cava imaged by intraoperative transesophageal echocardiography. Am Heart J. 1993;125:1185-7.

7. Mayer ED, Ruffmann K, Saggau W, Butzmann B, Bernhardt-Mayer K, Schatton N, et al. Ruptured aneurysms of the sinus of Valsalva. Ann Thorac Surg. 1986;42:81-5.

8. Takach TJ, Reul GJ, Duncan JM, Cooley DA, Livesay JJ, Ott DA, et al. Sinus of Valsalva aneurysm or fistula: management and outcome. Ann Thorac Surg. 1999;68:1573-7. 